\title{
Thickness-independent memory effect in ferroelectric liquid crystals
}

\author{
S. Kaur, A. K. Thakur, S. S. Bawa, and A. M. Biradar ${ }^{\mathrm{a})}$ \\ National Physical Laboratory, Dr. K. S. Krishnan Road, New Delhi-110012, India
}

(Received 22 September 2005; accepted 30 January 2006; published online 21 March 2006)

\begin{abstract}
Memory in ferroelectric liquid crystals (FLCs) is well known in the literature where thickness of the cell is less than the pitch value of the material. Here, we report a thickness independent memory in a class of FLCs called the de Vries electroclinic liquid crystals. Thickness independency of memory effect is observed by dielectric spectroscopy and texture observation. The memory observed in $\mathrm{Sm} \mathrm{C}^{*}$ phase of de Vries material is entirely different from conventional FLCs. In the former case, it is the inherent property of the material but in the latter it is dependent on the cell geometry. In de Vries material, it is probably the randomization that is playing a major role. (C) 2006 American Institute of Physics. [DOI: 10.1063/1.2186071]
\end{abstract}

Much of the research these days in the field of liquid crystals revolves around the ferroelectric liquid crystals (FLCs), which are quite promising in terms of high speed, better contrast, more durability and static memory. It was in 1974 that Meyer discovered ferroelectricity in smectic C phase composed of chiral molecules $\left(\mathrm{Sm} \mathrm{C}^{*}\right)$. ${ }^{1}$ The argument was lowering of $\mathrm{D}_{\infty}$ symmetry to $\mathrm{C}_{2}$ symmetry at the phase transition from $\mathrm{Sm} \mathrm{A}$ to $\mathrm{Sm} \mathrm{C} \mathrm{C}^{*}$; thus introducing spontaneous polarization in the molecules. This phenomenon was discovered for the first synthesized ferroelectric liquid crystal DOBAMBC. Since the discovery of ferroelectricity in DOBAMBC by Meyer et al. ${ }^{1}$ many other compounds have been synthesized which have $\mathrm{Sm} \mathrm{C}$, Sm H*, Sm I* phases or any combination of these.

A number of practical applications of FLCs such as displays, image control, spatial light modulator or memory devices make them popular in today's world. But these too come with respective constraints. One has to work with constraints of thickness such as in surface stabilized ferroelectric liquid crystal (SSFLC) geometry. Memory in SSFLC cells is dependent on the pitch of the material. ${ }^{2}$ SSFLC cells are made such that the thickness of the cell is less than the pitch of the material used. But in this letter we report memory in a special type of FLCs having wide and de Vries Sm A phase, which is thickness independent, i.e., memory is found irrespective of any thickness constraints in $\mathrm{Sm} \mathrm{C} \mathrm{C}^{*}$ phase of this ferroelectric liquid crystal.

These ferroelectric liquid crystals have a wide $\mathrm{Sm} \mathrm{A}$ phase and a high electroclinic coefficient $C_{E}=d \theta / d E$. It was the electroclinic effect based on the symmetry arguments of Garoff and Meyer. ${ }^{3,4}$ It was predicted by Meyer et al. that on applying electric field parallel to the smectic layers, the transverse component of the molecular polarization gives a nonzero value and thus the electroclinic effect. The formation of chevrons which are a folding instability of the smectic layer structure at the transition from $\mathrm{Sm} \mathrm{A}$ to $\mathrm{Sm} \mathrm{C}$ phase leads to poor contrast. ${ }^{5}$ This reduces the switching angle and considerably degrades the SSFLC geometry. A better approach is to select $\mathrm{Sm} \mathrm{C} \mathrm{C}^{*}-\mathrm{Sm}$ A phase material where there is no layer shrinkage. A number of materials were reported which exhibited little or no shrinkage of the smectic

${ }^{a}$ Author to whom correspondence should be addressed; electronic mail: abiradar@mail.nplindia.ernet.in layer spacing in $\mathrm{Sm} \mathrm{C}^{*}$ phase. It was in such materials that electroclinic effect was later found where the Sm A phase was tilted. Although the molecules are tilted in Sm A phase but the individual layer polarization is zero due to randomization of tilt in each layer. ${ }^{6-10}$ These are the de Vries liquid crystals reported by Diele, Brand, and Sackmann ${ }^{11}$ and later explained by de Vries in $1974 .^{12}$

It is in one of the de Vries FLC material that we report a thickness independent memory state. Though a lot of theoretical and experimental data have been reported about its dielectric and electro-optical studies, no light has been thrown on the behavior of the memory characteristics in $\mathrm{Sm} \mathrm{C}^{*}$ phase. The material has been studied in both thin as well as thick samples for memory response. Dielectric response along with the texture observation concludes that memory in the studied material is irrespective of the thickness of the sample cell. Though investigation at this stage does not reveal the exact mechanism behind the memory in these materials, probably on application of bias field the molecules get clamped to one position and do not come back to their original scattering state due to electrically induced inertia of the molecules.

In this letter, the ferroelectric liquid crystal 764E $(\mathrm{BDH}$, England) has been investigated for its memory effect. Cells for this study were made from indium tin oxide (ITO), coated on $\lambda / 2$ glass substrates. Homogeneously aligned cells of $20 \mu \mathrm{m}$ were filled with the material having the following phase sequence:

$$
\begin{aligned}
& \text { Cryst. ? Sm-C* }\left(28{ }^{\circ} \mathrm{C}\right) \mathrm{Sm}-\mathrm{A}\left(73^{\circ} \mathrm{C}\right) N \\
& \left(89-92{ }^{\circ} \mathrm{C}\right) \text { Iso. }
\end{aligned}
$$

The dielectric measurements were carried out using computer controlled HP 4192A impedance analyzer in the frequency range of $50 \mathrm{~Hz}-10 \mathrm{MHz}$. The texture was observed using polarizing microscope Carl Zeiss (Axioskop 40) and Linkam THMS 600 Hot stage.

In the present investigation, both thin as well as thick cells in the range of $1-20 \mu \mathrm{m}$ have been studied for the observance of memory in BDH 764E, which is a de Vries material. ${ }^{13}$ As far as surface stabilized ferroelectric liquid crystal geometry is concerned, the sample thickness is less than the pitch of the material. Due to surface stabilization one gets memory in a particular state. On the contrary, the concerned de Vries material has a pitch probably in the low 

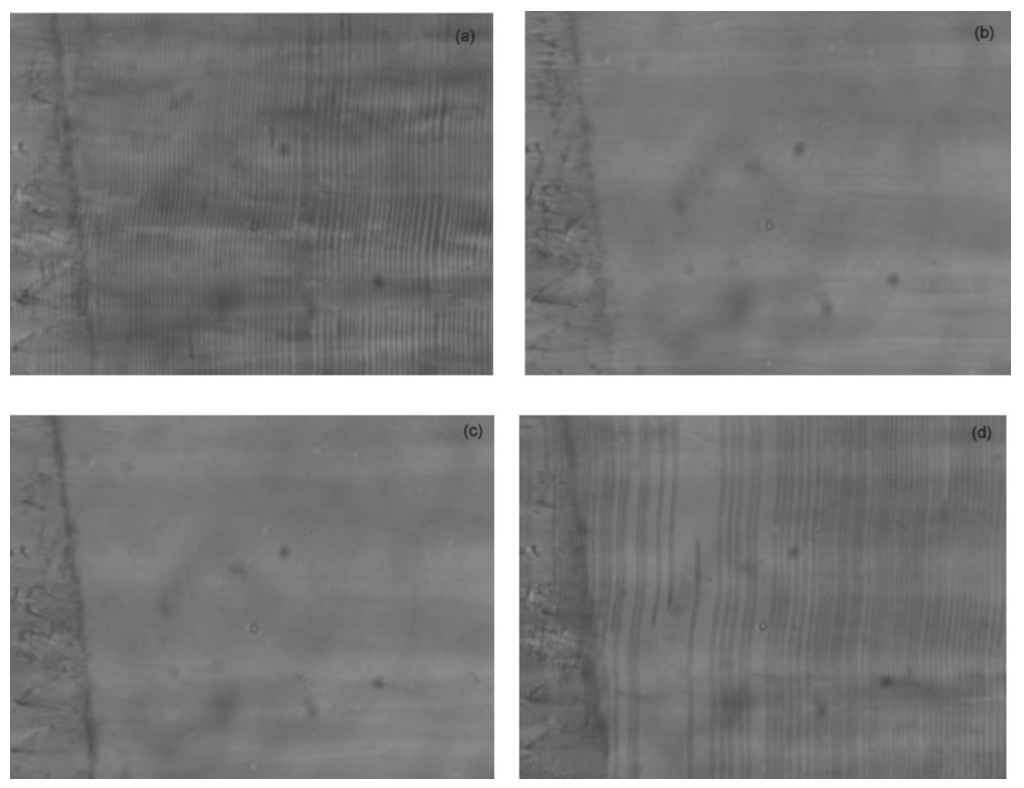

FIG. 1. Optical micrograph of electroclinic liquid crystal in Sm C $\mathrm{C}^{*}$ phase of 20 - $\mu$ m-thick cell at $14{ }^{\circ} \mathrm{C}$ at (a) $0 \mathrm{~V}$, (b) $30 \mathrm{~V}$ bias, (c) 5 min after removal of bias, (d) $1 / 2 \mathrm{~h}$ after removal of bias. The magnification is $50 \times$ in all the micrographs. value region around $2 \mu \mathrm{m}$. Pitch measurement was done in a well-aligned thick cell and found to be around $2 \mu \mathrm{m}$, in deep $\mathrm{Sm} \mathrm{C}^{*}$ phase. One can clearly see from the various methods that memory is found irrespective of the thickness of the sample.

Memory found in thick cells as well as thin cells was confirmed by observing the texture in deep $\mathrm{Sm} \mathrm{C}^{*}$ phase by the application of alternate $0 \mathrm{~V}$ bias after $30 \mathrm{~V}$ bias. One gets a clear picture of the memory in $\mathrm{Sm} \mathrm{C}^{*}$ phase. It can be visualized from Fig. 1(a) that dechiralization lines are present in a virgin cell in deep $\mathrm{Sm} \mathrm{C}^{*}$ phase. This is a picture at $0 \mathrm{~V}$ (i.e., no bias condition) at $14{ }^{\circ} \mathrm{C}$ in deep $\mathrm{Sm} \mathrm{C}{ }^{*}$ phase. On application of $30 \mathrm{~V}$ bias for $2-3 \mathrm{~min}$ at the same temperature, the dechiralization lines completely vanish as shown in Fig. 1(b). It clearly shows that the cell is in one of the stable states.

Now when the bias has been removed, where are the dechiralization lines which are a characteristic of the $0 \mathrm{~V}$ state? We find no such lines in Fig. 1(c). In fact, Fig. 1(c) very much resembles Fig. 1(b) in which bias is being continuously applied. Figure 1(d) shows the reappearance of dechiralization lines after an interval of nearly half an hour. One can say that the molecules have been clamped on to one side due to electrically induced inertia. The data presented are of thick cells of $20 \mu \mathrm{m}$. It is worth pointing out here that thin cells of $1 \mu \mathrm{m}$ thickness exhibit even better memory and that also for longer duration of time $(>12 \mathrm{~h})$. The optical response in thin as well as thick cells also reveals the same observation. Further investigation is still going on to find the basic mechanism which is causing the molecules to remain in the constrained state even after removal of the bias.

The dielectric tool is a strong evidence of the existence of memory in both thin as well as thick cells. Figure 2 showed the dielectric response in a $20 \mu \mathrm{m}$ cell. At $0 \mathrm{~V}$ bias the permittivity is quite high due to the phason mode at such low temperature, i.e., in deep $\mathrm{Sm} \mathrm{C}^{*}$ phase. A $30 \mathrm{~V}$ bias suppresses the fluctuation and the permittivity becomes low as seen in Fig. 2. But application of a $0 \mathrm{~V}$ bias again should have brought the permittivity to rise but does that happen? No such behavior was found; instead a low permittivity showed that the fluctuations were still suppressed as if bias is still being applied. Thus implying that the state was in Downloaded 09 May 2007 to 202.141.140.34. Redistribution subject memory state. The cell was in a memory state after removal of $30 \mathrm{~V}$ bias as $0 \mathrm{~V}$ bias permittivity matched with the $30 \mathrm{~V}$ value. Thin cells also showed similar dielectric response. One could conclude from these results that memory is found irrespective of any thickness constraints.

The observance of memory in conditions where there is no chance of finding the memory raises many questions about its structure, symmetry, and pitch of the material in the $\mathrm{Sm} \mathrm{C}^{*}$ phase. As it is a de Vries material, ${ }^{13}$ there is randomization near the transition temperature and in Sm A phase which has been explained by de Vries and many others. 6,14 But probably the randomization might not appear abruptly near the transition temperature; it might be that it is present even in $\mathrm{Sm} \mathrm{C}^{*}$ phase but the explanation of the symmetry of $\mathrm{Sm} \mathrm{C}^{*}$ phase and this expected randomization of molecules need critical study using group theory. If one studies the tilt of the studied material, it shows a constant value near the transition temperature at $5 \mathrm{~V}$ bias. There is no discontinuous jump at the transition temperature. ${ }^{15}$ The tilt behavior reveals that it is a nonlayer shrinkage material and does not even

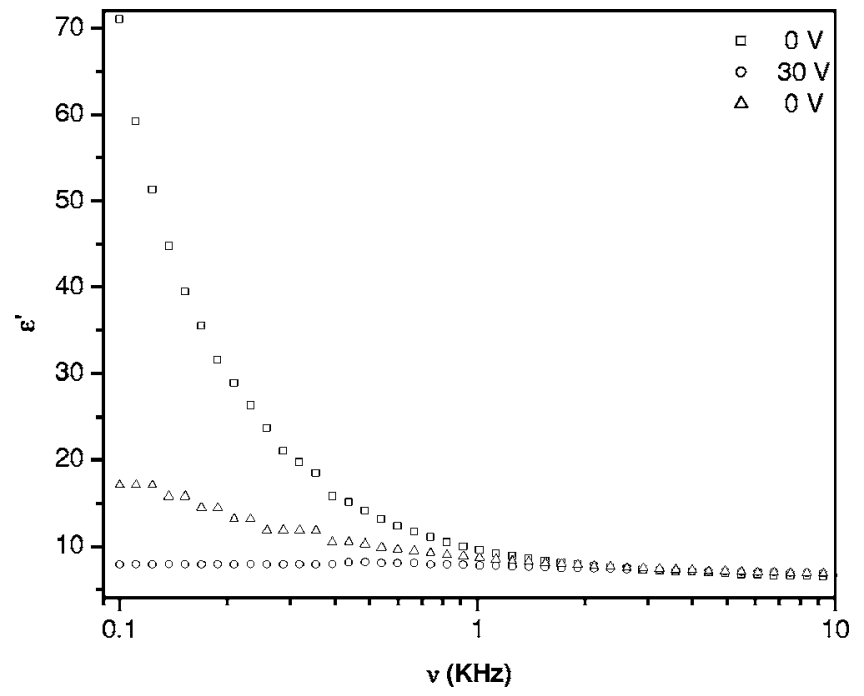

FIG. 2. Dielectric response as a function of frequency of electroclinic liquid crystal BDH 764E in $\mathrm{Sm} \mathrm{C}^{*}$ phase of 20 - $\mu \mathrm{m}$-thick cell at $14{ }^{\circ} \mathrm{C}$ at $0 \mathrm{~V}$ (open square), $30 \mathrm{~V}$ bias [open circle) and $0 \mathrm{~V}$ again (open triangle).

to AlP license or copyright, see http://apl.aip.org/apl/copyright.jsp 
follow the Curie-Weiss law, which has already been reported by us. ${ }^{13}$ As tilt is continuous near the transition temperature and there is no abrupt change in its behavior from $\mathrm{Sm} \mathrm{C}^{*}$ to $\mathrm{Sm}$ A phase, then randomization of the molecules is a chance that is occurring in $\mathrm{Sm} \mathrm{C}^{*}$ phase. Though the symmetry of the molecules in Sm $\mathrm{C}^{*}$ phase is still not clear but a distinct possibility of interplay of $\mathrm{C}_{2}$ and $\mathrm{D}_{\infty}$ symmetry that could contribute to some new kind of symmetry may not be ruled out. If the molecules are in random state in $\mathrm{Sm} \mathrm{C}$ phase then on application of bias they get directed to one particular state and remain there in the constrained state due to electrically induced inertia which the bias effect had left on the molecules. Moreover, the pitch of the material found is very small, which probably erases the thickness constraint. On the contrary, the SSFLC sample cells exhibit memory only due to the surface geometry or, in other words, surface stabilization accounts for the memory in SSFLC. But here in the ferroelectric de Vries liquid crystals memory is an inherent property of the material and the greatest advantage is that it does not depend on the geometry of the cell.

Finally, we can conclude on a short note that de Vries memory cells are much better than SSFLC memory cells as they do not involve the thickness constraint. Moreover, due to nonlayer shrinkage there are chevron free structures that exhibit a better contrast. The basic mechanism is still to be exactly unfolded but probably randomization is playing a major role in the memory of these cells.
The authors sincerely thank Dr. Vikram Kumar, Director, National Physical Laboratory, for continuous encouragement and interest in this work. The authors are thankful to DST, New Delhi, for supporting this work under the Project No. SP/S2/M-04/2000. The authors (S.K. and A.K.T.) are thankful to CSIR, New Delhi, for financial assistance.

${ }^{1}$ R. B. Meyer, L. Liebert, L. Strzelecki, and P. Keller, J. Phys. 36, L-69 (1975).

${ }^{2}$ N. A. Clark and S. T. Lagerwall, Appl. Phys. Lett. 36, 899 (1980).

${ }^{3}$ S. Garoff and R. B. Meyer, Phys. Rev. Lett. 38, 848 (1977).

${ }^{4}$ S. Garoff and R. B. Meyer, Phys. Rev. A 19, 338 (1979).

${ }^{5}$ T. P. Rieker, N. A. Clark, G. S. Smith, D. S. Parmar, E. B. Sirota, and C. R. Safinya, Phys. Rev. Lett. 59, 2658 (1987).

${ }^{6}$ J. P. F Lagerwall and F. Giesselmann, Phys. Rev. E 66, 031703 (2002).

${ }^{7}$ J. Naciri, C. Carboni, and A. K. George, Liq. Cryst. 30, 219 (2003).

${ }^{8}$ M. Rossle, R. Zentel, J. P. F. Lagerwall, and F. Giesselmann, Liq. Cryst. 31, 883 (2004).

${ }^{9}$ A. Tang, D. Konovalov, J. Naciri, B. R. Ratna, and S. Sprunt, Phys. Rev. E 65, 010703 (2002).

${ }^{10}$ R. B. Meyer and R. A. Pelcovits, Phys. Rev. E 65, 061704 (2002).

${ }^{11}$ S. Diele, P. Brand, and H. Sackmann, Mol. Cryst. Liq. Cryst. 16, 105 (1972).

${ }^{12} \mathrm{~A}$. de Vries, in Abstracts of the Fifth International Liquid Crystal Conference, Stockholm, edited by G. H. Brown (ILC Society, Kent, OH, 1974), p. 150.

${ }^{13}$ S. Kaur, A. K. Thakur, A. Choudhary, S. S. Bawa, A. M. Biradar, and S. Annapoorni, Appl. Phys. Lett. 87, 102507 (2005).

${ }^{14}$ J. V. Selinger, P. J. Collings, and R. Shashidhar, Phys. Rev. E 64, 061705 (2001).

${ }^{15}$ A. K. Thakur, G. K. Chadha, S. Kaur, S. S. Bawa, and A. M. Biradar, J. Appl. Phys. 97, 113514 (2005). 